\title{
Microtechnologies for Studying Cell Biology in Physiologically-Relevant Microenvironments
}

\section{C.A. Simmons*}

* Department of Mechanical and Industrial Engineering and Institute of Biomaterials and Biomedical Engineering, University of Toronto, Toronto, ON, Canada M5S 3G8

Cells reside in three-dimensional, soft extracellular matrices where they interact with other cells and, in the case of cardiovascular and musculoskeletal tissues, are subjected to dynamic mechanical loading. However, in traditional cell culture platforms (e.g., microtiter well plates), cells are grown on rigid, static two-dimensional surfaces. Thus, current platforms for studying cardiovascular and musculoskeletal cell biology poorly represent the in vivo environment, which limits the novelty and translatability of the biological information they generate.

In this talk, I will describe some of the microtechnologies that we are developing to address these limitations. These microfluidic platforms are designed to allow precise control over the cellular microenvironment, including matrix stiffness and proteins, soluble proteins, cell-cell interactions, and biophysical forces. Examples include microfluidic channels to mimic the vascular microenvironment [1-4]; arrays of microactuators that can be used to stretch cells or compress threedimensional cell-seeded hydrogels [5-8]; and cell-based arrays with tunable matrix mechanical and biochemical properties [9]. Compared with standard culture platforms, these microsystems better mimic key components of the in vivo cellular mechanobiological microenvironment, offer more precise control over microenvironmental cues, and avoid some of the confounding factors associated with application of mechanical forces at the macroscale. They are also compatible with on-chip imaging and are highly parallelizable, and therefore can be used in high-content and high-throughput screening applications. Current applications include fundamental studies of cell-matrix and cell-cell interactions in mechanically active environments and screening of biomaterial properties for stem cell-based tissue regeneration.

\section{References}

[1] E.W.K. Young et al., Lab Chip 7 (2007) 1759.

[2] E.W.K. Young and C.A. Simmons, Lab Chip 10 (2010) 143.

[3] E.W.K. Young et al., Anal. Chem. 82 (2010) 808.

[4] S. Srigunapalan et al., Biomicrofluidics, in press.

[5] C. Moraes et al., Biomaterials 31 (2010) 577.

[6] C. Moraes et al., Lab Chip 21 (2010) 227.

[7] C. Moraes et al., J Visualized Experiments (2010) www.jove.com/index/Details.stp?ID=2224.

[8] C. Moraes et al., J Micromech Microeng, in press.

[9] W.L.K. Chen et al., Biomaterials 31 (2010) 2489.

[10] This research was supported by the Natural Science and Engineering Research Council of Canada, the Canadian Institutes of Health Research, and the Canada Research Chair in Mechanobiology (to C.A.S.). 